BULL. AUSTRAL. MATH. SOC.

VOL. 31 (1985), 45I-462.

\title{
AN EGOROV'S THEOREM FOR VECTOR FUNCTIONS
}

\section{P. Jimenez Guerra and Jose L. de Maria Gonzalez}

\begin{abstract}
In this paper some results of Egorov's theorem type are given for functions with values in locally convex spaces and Riesz's theorem is proved for functions taking values in a sequentially complete locally convex space.
\end{abstract}

In [1] necessary and sufficient conditions are given for a sequence of measurable real functions to be almost uniformly convergent. In generalizing to functions taking values in more general spaces, some problems appear arising from the well-known fact (see [14]) that the sum of two Borel measurable functions is not, in general, Borel measurable. Some results in this area are given in [11].

In this paper some results of Egorov's theorem type are given for functions taking values in locally convex spaces, and a proof of Riesz's theorem is given for functions taking values in sequentially complete local convex spaces. From these results some examples of spaces which have Egorov's finite property with respect to certain families of functions were obtained in [7].

Chi in [5] gives the definition of the integral for functions which have "Egorov's property" and Gilliam in [9] supposes implicitly Egorov's property in the definition of his integral and measurability, as it is referred by Blondia in [4]. Rodriguez-Salinas uses it in [13]:to move from

Received 12 December 1984.

Copyright Clearance Centre, Inc. Serial-fee code: 0004-9727/85 $\$ \mathrm{~A} 2.00+0.00$. 
simple functions to $\mu$-measurable functions.

Throughout this paper we will denote by $(X, \mu, \Sigma)$ a complete measure space, by $E$ a locally convex space and by $\left(p_{i}\right)_{i \in I}$ a family of seminorms which defines the topology of $E$. All functions used here will be $E$-valued and defined on $X$.

DEFINITION 1 . A sequence $\left(f_{n}\right)_{n \in \mathbb{N}}$ is said to satisfy the vanishing restriction with respect to $f$ if for every $\varepsilon>0$ there exists a family of natural numbers $\left\{n(\alpha, i):(\alpha, i) \in \mathbf{R}^{+} \times I\right\}$ such that

$$
\mu^{*}\left[\begin{array}{cl}
U_{(\alpha, i) \in \mathbf{R}^{+} \times I} & E_{n(\alpha, i), i}^{f} \\
f(\alpha)
\end{array}\right]<\varepsilon
$$

holds, $\mu^{*}$ being the outer measure associated with $\mu, \mathbf{R}^{+}$the set of positive real numbers and

$$
E_{n, i}^{f}(\alpha)=\bigcup_{i=n}^{+\infty} A_{k, i}^{f}(\alpha) \quad(n \in \mathbb{N})
$$

where

$$
A_{k, i}^{f}(\alpha)=\left\{x \in X: p_{i}\left(f_{k}(x)-f(x)\right)>\alpha\right\}
$$

Evidently this definition is independent of the selected family of seminorms $\left(p_{i}\right)_{i \in I}$.

PROPOSITION 2. A sequence $\left(f_{n}\right)$ converges almost uniformly to a fronction $f$ if and only if it satisfies the vanishing restriction with respect to $f$.

Proof. Let us suppose first that $\left(f_{n}\right)$ is almost uniformly convergent to $f$. Then, given $\varepsilon>0$, there exists ${ }_{\varepsilon} \in \Sigma$ such that $\mu\left(B_{\varepsilon}\right)<\varepsilon$ and $\left(f_{n}\right)$ converges uniformly to $f$ on $X-B_{\varepsilon}$. So, for every $\alpha>0$ and $i \in I$, there exists $n(\alpha, i) \in \mathbb{N}$ such that $p_{i}\left[f_{k}(x)-f(x)\right] \leq \alpha$ holds for all $k \geq n(\alpha, i)$ and $x \in X-B_{\varepsilon}$. Therefore

$$
{ }_{B}^{B} \supset \underset{(\alpha, i) \in \mathbf{R}^{+} \times I}{U} \underset{k=n(\alpha, i)}{U} A_{k, i}^{f}(\alpha),
$$




$$
\mu^{*}\left[\underset{(\alpha, i) \in \mathbf{R}^{+} \times I}{U} \underset{k=n(\alpha, i)}{U} A_{\left.k, i^{(}\right)}^{f}(\alpha)\right]<\varepsilon
$$

and the sequence $\left(f_{n}\right)$ satisfies the vanishing restriction with respect to $f$.

Conversely, if $\left(f_{n}\right)$ satisfies the vanishing restriction with respect to $f$, then for every $\varepsilon>0$ there exists a family $\left\{n(\alpha, i):(\alpha, i) \in \mathbf{R}^{+} \times I\right\} \subset \mathbf{N}$ which satisfies (1.1) and therefore there exists $B_{\varepsilon} \in \Sigma$ such that $\mu\left(B_{\varepsilon}\right)<\varepsilon$ and

$$
B_{\varepsilon} \supset \underset{(\alpha, i) \in \mathbb{R}^{+} \times I}{U} E_{n(\alpha, i), i^{(\alpha)}}^{f} \text {. }
$$

So, for every $\alpha>0$ and $i \in I, p_{i}\left[f_{n}(x)-f(x)\right] \leq \alpha$ holds for all $n \geq n(\alpha, i)$ and $x \in B_{\varepsilon}^{c}$ and consequently $\left(f_{n}\right)$ converges uniformly to $f$ on $X-B_{\varepsilon}$.

DEFINITION 3. We say that a sequence $\left(f_{n}\right)$ satisfies the strong finite restriction with respect to a function $f$, if there exists a family $\left\{n(\alpha, i):(\alpha, i) \in \mathbf{R}^{+} \times I\right\} \subset \mathbf{N}$ such that

$$
\mu^{*}\left[\begin{array}{ll}
U & E_{\left.n(\alpha, i), i^{(}\right)}^{f}
\end{array}\right]<+\infty
$$

holds.

In the same way, we will say that $\left(f_{n}\right)$ satisfies the finite restriction with respect to $f$, if for every $\alpha>0$ there exists a family $\{n(\alpha, i): i \in I\} \subset N$ such that

$$
\mu^{*}\left[\bigcup_{i \in I} E_{n(\alpha, i), i}^{f}(\alpha)\right]<+\infty .
$$

Evidently, the last definitions are independent of the selected family of seminorms $\left(p_{i}\right)_{i \in I}$ and if a sequence $\left(f_{n}\right)$ satisfies the vanishing restriction with respect to a function $f$, then it satisfies also the strong finite restriction with respect to $f$ and therefore, it also satisfies the finite restriction with respect to $f$. Later on, the 
converse assertions are proved under certain conditions.

DEFINITION 4. Let $M$ be a family of $E$-valued functions defined on $X$. The locally convex space $E$ is said to satisfy the finite Egorov's condition with respect to $M$ if and only if every sequence in $M$ which converges almost everywhere to a function, is almost uniformly convergent on every measurable set $A \in \Sigma$ of finite measure.

The following results (among others) are proved in [10].

(a) If the locally convex space $E$ is metrizable or LF, then $E$ satisfies the finite Egorov's condition with respect to the family of the $E$-valued functions defined on $X$ whose restriction to every measurable set of finite measure, is a $\mu$-measurable function in the sense of [13]. The same result holds in the case of $E$ being the locally convex sum of a family of Fréchet spaces.

(b) If $E$ is a metrizable or LF space having weight of null measure, then $E$ satisfies the finite Egorov's condition with respect to the family of the $E$-valued functions defined on $E$ whose restriction to every measurable set of finite measure, is Borel measurable.

(c) If $E$ is a Banach space and its dual $E^{\prime}$ has the Radon-Nykodim property then $(E, b w(E))$ satisfies the finite Egorov's condition with respect to the family of essentially separable $E$-valued functions defined on $X$ whose restriction to every measurable set of finite measure, is $\hat{b} w(E)$-Borel measurable.

THEOREM 5. Let us suppose that $E$ satisfies the finite Egorov's condition with respect to a family of functions $M$, then a sequence $\left(f_{n}\right) \subset M$ satisfies the vanishing restriction with respect to a function $f$ if and only if it satisfies the finite restriction with respect to $f$ and $\left(f_{n}\right)$ is almost everywhere convergent to $f$.

Proof. If $\left(f_{n}\right)$ converges almost everywhere to $f$ and it satisfies the finite restriction with respect to $f$, then given $\varepsilon>0$, for $\alpha=I / p$ with $p \in \mathbb{N}$, there exists a family $\{n(1 / p, i): i \in I\} \subset \mathbb{N}$ which satisfies

$$
\mu^{*}\left[\bigcup_{i \in I}^{U} \underset{k=n(I / p, i)}{+\infty} A_{k, i}^{f}(I / p)\right]<+\infty .
$$


From this is deduced the existence of a set $A(1 / p) \in \Sigma$, with $\mu(A(1 / p))<+\infty$, such that

$$
A(I / p) \supset \bigcup_{i \in I}^{U} \bigcup_{k=n(1 / p, i)}^{+\infty} A_{k, i}^{f}(1 / p)
$$

holds. If we consider the restrictions $f_{n} \mid A(1 / p)$ and $f \mid A(1 / p)$ it follows from the hypothesis that the sequence $\left(f_{n} \mid A(1 / p)\right)$ satisfies the vanishing restriction with respect to $f \mid A(I / p)$ and there exists a family $\left\{Z(B, i): B \in \mathbf{R}^{+}, i \in I\right\} \subset \mathbf{N}$ such that

$$
\mu^{*}\left[\left(\underset{(\beta, i) \in \mathbb{R}^{+} \times I}{U} \underset{k=Z(\beta, i)}{U} A_{k, i}^{f}(\beta)\right) \cap A(I / p)\right]<\varepsilon / 2^{p}
$$

and

$$
\mu^{*}\left[\underset{i \in I}{U} \underset{k=Z(I / p, i)}{U} A_{k, i^{(I / p)} \cap A(I / p)}^{U}\right]<\varepsilon / 2^{p} .
$$

Indeed

$$
\underset{i \in I \quad}{U} U_{k=t(I / p, i)}^{+\infty} A_{k, i}^{f}(1 / p) \subset \underset{i \in I}{U} \underset{k=Z(I / p, i)}{U} A_{k, i}^{f}(I / p),
$$

where $t(1 / p, i)=\max \{(1 / p, i), n(1 / p, i)\}$, and so

$$
\mu^{*}\left[\underset{i \in I}{U} \underset{k=t(1 / p, i)}{U} A_{k, i}^{f}(1 / p)\right]<\varepsilon / 2^{p}
$$

and

$$
\mu^{*}\left[\begin{array}{cc}
U & +\infty \\
(p, i) \in N \times I & k=t(1 / p, i)
\end{array} A_{k, i}^{f}(1 / p)\right]<\varepsilon .
$$

For every $\alpha>0$ let $p \in N$ be the smallest natural number such that $1 / p \leq \alpha$ and let us take $t(\alpha, i)=t(1 / p, i)$. We are going to prove that

$$
\begin{gathered}
\underset{(\alpha, i) \in \mathbb{R}^{+} \times I}{U} \underset{k=t(\alpha, i)}{U} A_{k, i}^{f}(\alpha)=\underset{(p, i) \in \mathbb{N} \times I}{U} \underset{k=t(I / p, i)}{U} A_{k, i}^{f}(I / p) . \\
\text { If } x \in \underset{(\alpha, i) \in \mathbb{R}^{+} \times I}{U} \underset{k=t(\alpha, i)}{U} A_{k, i}^{f}(\alpha) \text { then there exists } \alpha \in \mathbf{R}^{+},
\end{gathered}
$$


$i \in I$ and $k \geq t(\alpha, i)$ such that $p_{i}\left(f_{k}(x)-f(x)\right)>\alpha$. Let $p$ be the smallest natural number with $1 / p \leq \alpha$, then we have $t(\alpha, i)=t(1 / p, i)$, $p_{i}\left(f_{k}(x)-f(x)\right)>1 / p$ and

$$
x \in \underset{(p, i) \in \mathbb{N} \times I}{U} \underset{k=t(1 / p, i)}{U} A_{k, i}^{f}(I / p) .
$$

So the sequence $\left(f_{n}\right)$ satisfies the vanishing restriction with respect to $f$. The opposite implication is trivial.

COROLLARY 6. If the space $E$ satisfies the finite Egorov's condition with respect to a family of functions $M$, then a sequence $\left(f_{n}\right) \subset M$ satisfies the vanishing restriction with respect to $f$ if and only if $\left(f_{n}\right)$ satisfies the strong finite restriction with respect to $f$ and converges almost everywhere to $f$.

COROLLARY 7. Under the conditions and notation of the previous corolzary, if the sequence $\left(f_{n}\right)$ is almost everywhere convergent to $f$, then $\left(f_{n}\right)$ satisfies the strong finite restriction with respect to $f$ if and only if it satisfies the finite restriction with respect to $f$.

DEFINITION 8. A sequence $\left(f_{n}\right)$ satisfies the vanishing restriction if for every $\varepsilon>0$ there exists a family of natural numbers $\left\{n(\alpha, i):(\alpha, i) \in \mathbf{R}^{+} \times I\right\}$ such that

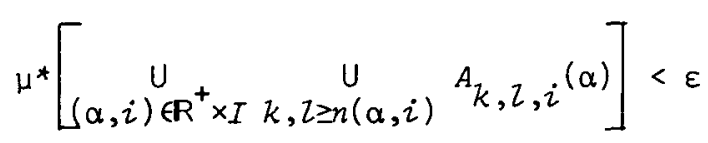

holds, where

$$
A_{k, Z, i}(\alpha)=\left\{x \in X: p_{i}\left(f_{k}(x)-f_{\imath}(x)\right)>\alpha\right\}
$$

$\left(f_{n}\right)$ is said to be a Cauchy sequence in measure if

$$
\lim _{k, Z \rightarrow+\infty} \mu^{*}\left[\bigcup_{i \in I}^{U} A_{k, Z, i}(\alpha)\right]=0
$$

for all $\alpha>0$. In the same way, we will say that $\left(f_{n}\right)$ is convergent in measure to $f$ when 


$$
\operatorname{Iim}_{n} \mu^{*}\left[\operatorname{U}_{i \in I} A_{n, i}^{f}(\alpha)\right]=0
$$

holds for every $\alpha>0$.

Finally we will say that $\left(f_{n}\right)$ is M-convergent to $f$ if for all $\alpha>0$ and $i \in I$ it is true that

(8.4) $\left\{x \in X: p_{i}\left(f_{n}(x)-f(x)\right)>\alpha\right\} \subset\left\{x \in X: p_{i}\left(f_{k}(x)-f(x)\right)>\alpha\right\}$

whenever $k \leq n$.

PROPOSITION 9. If the space $E$ is Hausdorff and sequentially complete and the sequence $\left(f_{n}\right)$ satisfies the vanishing restriction then there exists a function $f$ such that $\left(f_{n}\right)$ converges to $f$ almost uniformly.

Proof. Indeed, given $\varepsilon>0$ there exists a family $\left\{n(\alpha, i):(\alpha, i) \in \mathbb{R}^{+} \times I\right\} \subset N$ such that (8.1) is satisfied and therefore it is possible to find a set $B_{\varepsilon} \in \Sigma$ with $\mu\left(B_{\varepsilon}\right)<\varepsilon$ and

$$
X-B_{\varepsilon} \subset \bigcap_{(\alpha, i) \in \mathrm{R}^{+} \times I} \bigcap_{k, Z \geq n(\alpha, i)} A_{k, \tau, i}^{c}(\alpha)
$$

Then for every $\alpha>0$ and $i \in I$ there exists $n(\alpha, i) \in \mathbb{N}$ such that $p_{i}\left(f_{k}(x)-f_{l}(x)\right) \leq \alpha$ whenever $k, z \geq n(\alpha, i)$ and $x \in X-B_{\varepsilon}$. So there exists $g_{\varepsilon}(x)=\lim _{n} f_{n}(x)$ for all $x \in X-B_{\varepsilon}$ and evidently the sequence $\left(f_{n}\right)$ is almost uniformly convergent to the function

$$
f(x)= \begin{cases}0 & \text { if } x \in B_{0}, \\ g_{1 / n}(x) & \text { if } x \notin B_{1 / n},\end{cases}
$$

being $B_{0}=\bigcap_{n \in \mathbb{N}} B_{1 / n}$.

PROPOSITION 10. If $E$ is Hausdorff and sequentially complete and the sequence $\left(f_{n}\right)$ is convergent in measure to a function $f$ and satisfies the vanishing restriction then it is almost uniformly convergent to $f$. 
Proof. The existence of a function $g$ such that $\left(f_{n}\right)$ is almost uniformly convergent to $g$ follows from Proposition 9 . Let us prove that $f=g$ almost everywhere. Clearly

$$
\{x \in X: g(x) \neq f(x)\} \subset \bigcup_{n \in \mathbb{N}} \bigcup_{i \in I}\left\{x \in X: p_{i}(f(x)-g(x))>1 / n\right\}
$$

and

$$
\begin{aligned}
\bigcup_{i \in I}\left\{x \in X: p_{i}(f(x)-g(x))>1 / n\right\} & <\left[\underset{i \in I}{U}\left\{x \in X: p_{i}\left(f(x)-f_{k}(x)\right)>1 / 2 n\right\}\right] \\
\cup & {\left[\underset{i \in I}{U}\left\{x \in X: p_{i}\left(f_{k}(x)-g(x)\right)>1 / 2 n\right\}\right] }
\end{aligned}
$$

for all $k \in N$, from which we deduce that $\mu^{*}\{x \in X: g(x) \neq f(x)\}=0$.

PROPOSITION 11. If the sequence $\left(f_{n}\right)$ is M-convergent and converges in measure to a function $f$, then $\left(f_{n}\right)$ satisfies the vanishing restriction with respect to $f$.

Proof. In this case we have that

$$
\bigcup_{i \in I} \underset{k=n}{U} A_{k, i}^{f}(\alpha)=\bigcup_{i \in I} A_{n, i}^{f}(\alpha)
$$

for all $\alpha \in \mathbf{R}^{+}$and $n \in \mathbb{N}$, and since

$$
\lim _{n} \mu^{*}\left(\underset{i \in I}{U} A_{n, i}^{f}(\alpha)\right)=0
$$

for every $\alpha>0$, given $\alpha=1 / k$, with $k \in N$, there exists $n(1 / k) \in N$ such that

$$
\mu^{*}\left(\bigcup_{i \in I} A_{l, i}^{f}(I / k)\right]<\varepsilon / 2^{k}
$$

for all $l \geq n(1 / k)$ and so

$$
\mu^{*}\left(\bigcup_{k \in \mathbb{N}}^{U} \underset{i \in I}{U} A_{n(1 / k), i}^{f}(1 / k)\right)<\varepsilon,
$$

from which, in a similar manner to the proof of Theorem 5, we deduce that $\left(f_{n}\right)$ satisfies the vanishing restriction with respect to $f$.

THEOREM 12 (Riesz). Let $E$ be a Hausdorff and sequentially 
An Egorov's theorem

459

complete locally convex space and $\left(f_{n}\right)$ a Cauchy sequence in measure, then there exists a subsequence $\left(f_{n_{k}}\right)$ which satisfies the vanishing restriction and is almost uniformly convergent to a function $f$. Moreover, the sequence $\left(f_{n}\right)$ converges in measure to $f$.

Proof. Indeed, for all $\alpha>0$, we have that

$$
\lim _{k, Z} \mu^{*}\left(U_{i \in I}^{U} A_{k, Z, i}(\alpha)\right)=0 \text {, }
$$

from where follows the existence of a non-decreasing sequence $\left(n_{k}\right) \subset \mathrm{N}$ such that

$$
\mu^{*}\left(\bigcup_{i \in I} A_{h, Z, i}\left(1 / 2^{k}\right)\right)<1 / 2^{k}
$$

for all $h, \quad l \geq n_{k} \quad(k \in N)$.

$$
\begin{aligned}
& \text { Let us define } g_{k}=f_{n_{k}+1} \text { and } \\
& \qquad A_{k}=\bigcup_{i \in I}\left\{x \in X: p_{i}\left(g_{k+1}(x)-g_{k}(x)\right)>1 / 2^{k}\right\}
\end{aligned}
$$

for every $k \in N$. Then $\mu^{*}\left(A_{k}\right)<1 / 2^{k}$ and $\mu^{*}\left(F_{k}\right)<1 / 2^{k-1}$, where

$$
\begin{aligned}
f_{k}=\cup_{j=k}^{+\infty} A_{j}(k \in N) . & \text { If } h \geq Z \geq k \text { and } x k F_{k} \text { then } \\
p_{i}\left(g_{h}(x)-g_{\eta}(x)\right) & \leq p_{i}\left(g_{h}(x)-g_{h-1}(x)\right)+\ldots+p_{i}\left(g_{\eta+1}(x)-g_{\eta}(x)\right) \\
& \leq 1 / 2^{k-1}
\end{aligned}
$$

holds for all $i \in I$. If, for $l, h \in N, i \in I$ and $\alpha>0$,

$$
A_{i, h, i}(\alpha)=\left\{x \in X: p_{i}\left(g_{i}(x)-g_{h}(x)\right)>\alpha\right\}
$$

then

$$
\begin{array}{r}
\underset{i \in I h, Z \geq n}{\cup} A_{i, h, i}^{\prime}(\alpha) \subset F_{k} \\
\text { for all } n \geq k \text {, being } 1 / 2^{k-1} .
\end{array}
$$

https://doi.org/10.1017/S0004972700009412 Published online by Cambridge University Press 
Therefore, for $\alpha, \varepsilon>0$, let us consider $k_{1}(\alpha) \in \mathbb{N}$ such that $1 / 2^{k_{1}(\alpha)-1}<\min (\alpha, \varepsilon)$, then

$$
\bigcup_{i \in I} \bigcup_{h, l \geq n} A_{h, Z, i}^{\prime}(\alpha) \subset F_{k}
$$

and

$$
\mu^{*}\left(\bigcup_{i \in I} \underset{h, Z \geq n}{U} A_{h, Z, i}^{\prime}(\alpha)\right)<\varepsilon
$$

hold for all $n \geq k_{1}(\alpha)$.

So, given $\alpha=1 / p$, with $p \in \mathbb{N}$, there exists $k_{1}(1 / p) \in \mathbb{N}$ such that

$$
\mu^{*}\left(\bigcup_{i \in I} \underset{h, Z \geq k_{1}(I / p)}{U} A_{h, l, i}^{\prime}(I / p)\right)<\varepsilon / 2^{p}
$$

from which it follows immediately that

$$
\mu^{*}\left(\operatorname{viN}_{i \in I}^{U} \underset{h, Z \geq k_{I}(I / p)}{U} A_{h, Z, i}^{\prime}(1 / p)\right)<\varepsilon .
$$

Now, proceeding in the same way as in the proof of Theorem 5, we have that the sequence $\left(g_{n}\right)$ satisfies the vanishing restriction and so, from Proposition 9, the theorem follows.

LEMMA 13. Let $g$ be a non-negative real function (defined on $X$ ) with a finite distribution function (see [1], Definition 2.1). If $\left(f_{n}\right)$ and $f$ are such that $p_{i}:\left(f_{n}-f\right) \leq g$ almost everywhere for all $n \in \mathbb{N}$ and $i \in I$, then the sequence $\left(f_{n}\right)$ satisfies the finite restriction with respect to $f$.

Proof. It is immediate.

THEOREM 14. With the notation of Lemma 13, if $E$ satisfies the finite Egorov's condition with respect to a family of functions $M$, $\left(f_{n}\right) \subset M$ is almost everywhere convergent to $f$ and $p_{i}, f_{n} \leq g$ almost everywhere, then $\left(f_{n}\right)$ is almost uniformly convergent to $f$. 
Proof. This is an immediate consequence of Lemma 13.

\section{References}

[1] R.G. Bartle, "An extension of Egorov's theorem", Amer. Math. Monthly 87 (1980), 628-633.

[2] R.G. Bartle, "Properties equivalent to almost uniform convergence", Proceedings Conference Northerm Illinois University, 155-160 (Northern Illinois University, DeKalb, Illinois, 1980).

[3] C. Blondia, "Radon-Nikodym theorem for vector valued measures", Bull. Soc. Math. Belg. Ser. B 23 (1981), 231-249.

[4] C. Blondia, "Integration in locally convex spaces", Simon Stevin 55 (1981), 81-102.

[5] G.Y. Chi, "On the Radon-Nikodym theorem in locally convex spaces", Measure theory, 199-209 (Proc. Conf. Oberwolfach, 1975. Lecture lotes in Mathematics, 541. Springer-Verlag, Berlin, Heidelberg, New York, 1976).

[6] Dobrakov, "On integration in Banach spaces, I", Czechoslovak Math. J. 20 (1970), 511-536.

[7] Dobrakov, "On integration in Banach spaces, II", Caechoslovak Math. J. 20 (1970), 680-695.

[8] Nelson Dunford and Jacob T. Schwartz, Linear operators, Part II (Interscience [John Wiley \& Sons], New York, London, 1963).

[9] Gilliam, "On integration and the Radon-Nikodym theorem in quasi complete locally convex topological vector spaces", J. Reine Angew. Math. 292 (1977), 125-137.

[10] Jose L. de Maria Gonzalez, "A characterization theorem for locally convex space valued functions", IlZinois J. Math. 28 (1984), 592-596.

[11] José L. de María Gonzalez, "Un théorème de Marczewski-Sikorski pour les espaces LF", Actualités Mathématiques, 407-410 (GauthierVillars, Paris, 1982). 
[12] Jose L. de Maria Gonzalez, "Extensiones al teorema de Egorov" (Tesis Doctoral. Universidad Complutense de Madrid, Madrid, 1981).

[13] B. Rodriguez-Sal inas, "Integracion de funciones con valores en un espacio localmente convexo", Rev. Real Acad. Cienc. Exact. Fis. Natur. Madrid 63 (1979), 361-387.

[14] A.A. Stone, "Topology and measure", Measure theory, 43-48 (Proc. Conf. Oberwolfach, 1975. Lecture Notes in Mathematics, 541. Springer-Verlag, Berlin, Heidelberg, New York, 1976).

Departmento de Teoria de Functiones, Facultad de Ciencias Matematicas,

Ciudad Universitaria,

Madrid 3, Spain. 